\title{
The Impact of Dynamic Range on Open-Loop Power Control Error
}

\author{
Ai Yu and Jiangzhou Wang \\ Department of Electrical and Electronic Engineering \\ The University of Hong Kong, \\ Pokfulam Road, Hong Kong \\ Tel: 852-2859 2169 \\ Fax: 852-2559 8738 \\ E-mail: ayu@eee.hku.hk
}

\begin{abstract}
Direct Sequence Code Division Multiple Access (DS-CDMA) techniques have received a great deal of attention for current and future communication systems. One of the major considerations in implementing a DS-CDMA system is the necessity for accurate power control to ensure adequate quality-of-service and capacity. This paper investigates the measurement error of an open-loop power control algorithm under transmit power limitation. The power control error is analysed and numerically computed.
\end{abstract}

\section{Introduction}

As well known, a DS/CDMA system is susceptible to near-far interference, which occurs when the base station input includes one or more other CDMA signals that are stronger than the desired signal. The near-far effect can be reduced by adjusting the transmitted power of all mobile users so that the base station gets the same power from the received signal of each transmission. Two types of power control are often considered: closed-loop power control and open-loop power control. In a closed-loop power control, according to the received signal power at a base station, the base station sends a command to a mobile to adjust the transmit power of the mobile. However, in an open-loop power control, a mobile user adjusts its transmit power according to its received power in downlink. In this paper, an adaptive open-loop power control algorithm [1] is adopted. The algorithm produces an estimate of the received power at the mobile by averaging squared outputs of the correlator.

The open-loop power control error usually results from the factors such as the accuracy of power measurement at a mobile, the dynamic ranges of the transmit power of mobiles and the loop delay. However, in [1], only measurement error is concentrated. As we know, for an adaptive algorithm, a larger dynamic range is required for getting a good performance. In this paper, the impact of dynamic range on open-loop power control error is investigated.

The paper is organized as follows. The downlink system model is given in Section II. In section III, the open-loop power control error with upper bound transmit power limitation is studied. Numerical results are given in Section IV. Conclusions will be given in the last section.

\section{System Model}

Neglecting the white noise on the downlink, the waveforms received by each mobile is composed of the desired signal, a number of interfering signals from the base station of the cell of interest and the adjacent-cell base stations.

Assuming that all spreading codes of each cell are orthogonal and that the downlink transmissions of a cell are synchronized, the intracell interference can be ignored because of the same transmission delay. However, the interfering signals from adjacent-cell base stations cannot be assumed to be synchronized with the desired signal because of different transmission delays. This interference becomes significant when a mobile moves towards the boundary of its cell.

In order to study the downlink adjacent-cell interference, two layers of cells are considered (see Fig.1). Each user is assumed to be located independently of all the other users and uniformly distributed over the area of the cell. The location of a reference user in the first cell (the cell of interest) is $\left(r_{13}, \theta_{11}\right)$, where $r_{11}$ and $\theta_{11}$ stand for the distance and angle of the mobile from its base station. The distance between the base station of the $i$ th adjacent cell and the reference mobile of the first cell is given by

$r_{i 1}=\sqrt{d_{i 1}^{2}+r_{11}^{2}-2 d_{i 1} r_{11} \cos \theta_{i 1}}, \quad i=2,3, \ldots, 19$

where $d_{i 1}$ is the distance between the first base station and the $i$ th base station, and 


$$
d_{i 1}= \begin{cases}\sqrt{3} R, & i=2,3, \ldots, 7 \\ 2 \sqrt{3} R, & i=8,10,12,14,16,18 \\ 3 R, & i=9,11,13,15,17,19\end{cases}
$$

where $R$ stands for the radius of the hexagonal cell. In (1), $\theta_{i 1}$ is the angle between $d_{i 1}$ and $r_{11}$. The relationship between $\theta_{i 1}$ and $\theta_{11}$ for various values of $i$ is given by

$$
\cos \left(\theta_{i 1}\right)=\left\{\begin{array}{ll}
\cos \left[\theta_{11}+(i+2) \pi / 3\right], & 2 \leq i \leq 7 \\
\cos \left(\theta_{11}+i \pi / 6\right), & 8 \leq i \leq 19
\end{array} .\right.
$$

The channel is modelled as a frequency nonselective channel with Rayleigh statistics and lognormally distributed shadowing. The mobile receiver is a conventional coherent receiver. Assuming that there are $K$ active users for each cell, the received signal at mobile user1 (reference user) can be written as

$$
\begin{gathered}
r\left(\left.t\right|_{r_{i 1}, \theta_{11}}\right)=\sum_{i=1}^{|l|} \sum_{k=1}^{K} \sqrt{2 P_{R} \xi_{i k} r_{i 1}^{-\gamma}} a_{i}(t) e^{\zeta_{i}(t)} c_{i k}\left(t-\tau_{i}\right) \\
\cdot b_{i k}\left(t-\tau_{i}\right) \cos \left(2 \pi f_{0} t-\phi_{i}\right)
\end{gathered}
$$

where $P_{R}$ is the transmitted power of the base station when the reference user is located at a cell vertex $\left(r_{11}=R\right)$, and $\xi_{i k}\left(0<\xi_{i k} \leq 1\right)$ is the downlink power adjustment factor (or power control function) and $\xi_{11}=1$ when $r_{11}=R . r_{i 1}^{-\gamma}$ represents the propagation path loss from the $i$ th base station to the reference user and $\gamma$ may take values between two and four. $a_{i}(t)$ is the Rayleigh distributed random variable with unitary power, i.e. $E\left[a_{i}^{2}(t)\right]=1 . \quad e^{\zeta_{i}(t)}$ is the log-normal shadowing with random variable $\zeta_{i}(t)$, taking zero mean and a typical variance of $8 \mathrm{~dB}$. The single subscript of $a_{i}(t)$ and $\zeta_{i}(t)$ means that all the signals transmitted by each base station propagate on the same path, and undergo the same fading and shadowing. $\quad c_{i k}(t)$ and $b_{i k}(t)$ represent the spreading sequence with chip duration $T_{c}$ and the binary data sequence with duration $T$ of user $k$ of cell $i$, respectively. $\tau_{i}$ and $\phi_{i}$ are the corresponding time delay and phase, respectively. Note that $\tau_{l}$ and $\phi_{i}$ are the same for all users of cell $i$, because of the synchronous downlink transmission. $f_{0}$ is the CDMA carrier frequency on downlink.

Assuming $\tau_{1}=\phi_{1}=0$ for the first cell, the output of despread/demodulator at time $l T$, is given by

$$
Z\left(\left.I T\right|_{r_{1}, \theta_{11}}\right)=\frac{1}{T} \int_{(l-1) T}^{(T T} r\left(\left.t\right|_{r_{1}, \theta_{11}}\right) \cdot 2 c_{11}(t) \cos \left(2 \pi f_{0} t\right) d t
$$

where $2 \cos \left(2 \pi f_{0} t\right)$ and $c_{11}(t)$ are the recover carrier and the spreading sequence of the reference user. Since high frequency terms are removed by the lowpass filter following the mixer of the demodulator, the above expression reduces to $Z\left(\left.l T\right|_{r_{1}, b_{11}}\right) \approx \sqrt{2 P_{R} \xi_{11} r_{11}^{-\gamma}} a_{1}(l T) e^{\zeta_{1}(I T)} b_{11}(l T)+I(l T)$.

Note that, in the following, " $l T$ " is replaced by " $l$ " for simple notation. The first term on the right hand right of (6) is the desired component. The second term on the right hand side of (6) is the adjacent-cell interference, and is expressed as

$$
I(l)=\sum_{i=2}^{19} a_{i}(l) e^{\zeta_{i}(l)} \sum_{k=1}^{K} \sqrt{2 P_{R} \xi_{i k} r_{i 1}^{-\gamma}} I_{i, l}(l) \cos \phi_{l}
$$

where

$$
I_{i, 1}(l)=\frac{1}{T} \int_{(l-1) r}^{i r} b_{i k}\left(t-\tau_{i}\right) c_{i k}\left(t-\tau_{i}\right) c_{11}(t) d t .
$$

When $K$ is large, $I_{i, 1}(l)$ can be approximated by a Gaussian random variable with the variance of $\operatorname{var}\left[I_{i, 1}(l)\right]=2 /(3 N)$. Then, the variance of $I(l)$ is given by

$$
\sigma_{I}^{2}=\frac{2 K}{3 N} e^{2 \sigma_{s i}^{2}} \sum_{i=2}^{19} P_{R} E\left[\xi_{i k}\right] r_{i 1}^{-\gamma}
$$

where $E\left[\xi_{i k}\right]$ stands for the mean of downlink power adjustment factor. Following the optimum downlink power allocation scheme [2]. $\xi_{11}$ is given by

$$
\xi_{11} \approx \frac{\sum_{i=2}^{19}\left(r_{i 1} / r_{11}\right)^{-\gamma}}{\left.\sum_{i=2}^{19}\left(r_{i 1} / R\right)^{-\gamma}\right|_{\left(r_{1}=R, \theta_{11}=\pi / 6\right)}} .
$$

Assuming that the position of each user is uniformly distributed in a cell, i.e.,

$$
f\left(r_{11}, \theta_{11}\right)=\frac{1}{\pi R^{2}}, 0 \leq r_{11} \leq R, \quad 0 \leq \theta_{11} \leq 2 \pi,
$$

the expectation of $\xi_{i k}$ is obtained by double integration of (10),

$$
\begin{aligned}
E\left[\xi_{i k}\right] & =E\left[\xi_{11}\right] \\
& =\frac{1}{\pi R^{2}} \int_{11}^{2 \pi} \int_{0}^{R} \xi_{11} r_{11} d r_{11} d \theta_{11}=0.3189 .
\end{aligned}
$$

\section{Power Control Error}

Similar to [1], this paper employs adaptive open-loop power control algorithm. In this algorithm, the received signal power at the mobile is estimated by averaging the output square of a correlator. The estimate at time $L T$ is given by averaging the square of (6),

$$
\hat{S}^{2}(L)=\frac{1}{L} \sum_{i=1}^{L} Z^{2}\left(\left.l\right|_{r_{1}, \theta_{11}}\right)
$$




$$
\begin{aligned}
=\frac{1}{L} \sum_{l=1}^{L} 2 P_{R} \xi_{11} r_{11}^{-\gamma} a_{1}^{2}(l) e^{2 \xi_{1}(l)} \\
+\frac{2}{L} \sum_{l=1}^{L} \sqrt{2 P_{R} \xi_{11} r_{11}^{-\gamma}} a_{1}(l) e^{\xi_{1}(l)} I(l) \\
\quad+\frac{1}{L} \sum_{l=1}^{L} I^{2}(l) .
\end{aligned}
$$

The mobile transmitter uses this estimate to adjust its transmit power, which is inversely proportional to the estimated power, i.e. $P_{t}=1 /\left(C \cdot \hat{S}^{2}(L)\right)$, where $C$ is a weighted constant.

According to the above power control scheme, the transmitted power in a CDMA system can be adjusted to any desired value. However, in practice, the transmitter power is limited with an upper bound. If $P_{t m a x}$ is the maximum transmitted power, the transmitted power is revised to

$$
P_{t}=\min \left\{l /\left(C \cdot \hat{S}^{2}(L)\right), \quad P_{t \max }\right\} .
$$

Neglecting the loop delay, the desired signal component after despreading at the base station at the time $L T$, is given by

$$
\begin{aligned}
Z_{1}(L) & =\sqrt{2 P_{1} r_{11}^{-\gamma}} a_{1}^{\prime}(L) e^{\zeta_{1}(L)} b_{11}^{\prime}(L) \\
& =\sqrt{2} P_{e r r} a_{1}^{\prime}(L) b_{11}^{\prime}(L)
\end{aligned}
$$

where $a^{\prime}(L), e^{\zeta_{1}(L)}$ and $b_{11}^{\prime}(l)$ mean the Rayleigh fading, lognormal distributed shadowing and data bit, respectively, in the uplink. Notice that the shadowing terms on both up and down links are the same, but the fading terms on the two links are different. $P_{e r r}$ is the power control error, defined as

$$
\begin{aligned}
P_{e r r} & =\sqrt{\min \left\{1 /\left(C \cdot \hat{S}^{2}(L)\right), P_{\text {tmax }}\right\} r_{11}^{-\gamma} e^{2 \zeta_{1}(L)}} \\
& =\sqrt{\min \left\{r_{11}^{-\gamma} e^{2 \zeta_{1}(L)} /\left(C \hat{S}^{2}(L)\right), P_{\text {tmax }} r_{11}^{-\gamma} e^{2 \zeta_{1}(L)}\right\} .}
\end{aligned}
$$

When there is no upper bound transmit power limitation, it is assumed the received power control error has a log-normal distribution, i.e. $r_{11}^{-\gamma / 2} e^{\zeta_{1}(L)} / \sqrt{\operatorname{CS}^{2}(L)}=e^{y}$. The mean and standard derivation of $y$ can be derived as [1], i.e.

$$
\bar{y}=\frac{1}{2} \ln \left(\sqrt{V} / C U^{2}\right)
$$

and

$$
\sigma_{y}=\frac{1}{2} \sqrt{\ln \left(V / U^{2}\right)} .
$$

By choosing $C$ appropriately, i.e. $C=\sqrt{V} / U^{2}$, the mean of $y$ can be set to zero $(\bar{y}=0)$, corresponding to an unbiased estimate in absence of transmit power limitation. In (17) and (18), $U$ and $V$ is given by

$$
U=\frac{2 P_{R} \xi_{11}}{L} \sum_{l=1}^{L} \exp \left(4 \sigma_{\zeta_{2}}^{2}-4 C_{\zeta_{1}}((l-L) T)\right)
$$

$$
+\frac{1}{r_{11}^{-\gamma}} e^{2 \sigma_{\zeta_{1}}^{2}} \sigma_{I}^{2}
$$

and

$$
\begin{aligned}
V= & \frac{4 P_{R}^{2} \xi_{11}^{2}}{L^{2}} \sum_{l_{1}=1}^{L} \sum_{l_{2}=1}^{L}\left(1+\rho^{2}\left(l_{1}-l_{2}\right)\right) \cdot \exp \left[12 \sigma_{\zeta_{1}}^{2}+4 C_{\zeta_{1}}\left(\left(l_{1}-l_{2}\right) T\right)\right. \\
& \left.-8 C_{\zeta_{1}}\left(\left(l_{1}-L\right) T\right)-8 C_{\zeta_{1}}\left(\left(l_{2}-L\right) T\right)\right] \\
& +\frac{8 P_{R} \xi_{11}}{L^{2} r_{11}^{-\gamma}} \sigma_{I}^{2} \sum_{l=1}^{L} \exp \left(10 \sigma_{\zeta_{1}}^{2}-8 C_{\zeta_{1}}((l-L) T)\right) \\
& +\left(1+\frac{2}{L}\right) \frac{\sigma_{I}^{4}}{r_{11}^{-2 \gamma}} e^{8 \sigma_{\zeta_{1}}^{2}} \\
& +\frac{4 P_{R} \xi_{11}}{L r_{11}^{-\gamma}} \sigma_{I}^{2} \sum_{l=1}^{L} \exp \left(10 \sigma_{\zeta_{1}}^{2}-8 C_{\zeta_{1}}((l-L) T)\right) .(20)
\end{aligned}
$$

where $C_{\zeta_{1}}(\tau)$ is the autocovariance function of $\zeta_{!}(t)$, given by [3]

$$
\begin{aligned}
C_{\zeta_{1}}(\tau) & =E\left[\zeta_{1}(t) \zeta_{1}(t+\tau)\right]-E^{2}\left[\zeta_{1}(t)\right] \\
& =\sigma_{\zeta_{1}}^{2} e^{-v|\tau| / D}
\end{aligned}
$$

where $v$ is a mobile speed, $D$ represents the correlation distance and has been measured as hundreds of meters for conventional terrestrial cells, and tens of meters for terrestrial microcells. In $(20), \rho(\tau)$ is the normalized autocovariance function of the Rayleigh process, given by [4]

$$
\rho(\tau)=J_{0}\left(2 \pi f_{d}|\tau|\right)
$$

where $f_{d}$ is a maximum Doppler frequency or fading rate, given by $f_{d}=v / \lambda$, where $\lambda$ is a carrier wavelength. $J_{0}(x)$ is a Bessel function of the first kind of zeroth order.

We defined the mean and standard derivation of $e^{y}$ as the mean and standard derivation of power control error under the case there is no upper bound transmit power limitation. They are given by

$$
E\left[e^{y}\right]=e^{\sigma_{y}^{2} / 2}
$$

and

$$
\sigma_{e^{y}}=e^{2 \sigma_{y}^{2}}-e^{\sigma_{y}^{2}} .
$$

Because $e^{y}$ is log-normal distributed, from [5], the $p d f$ of $e^{2 y}$ is given by

$$
f_{e^{2 y}}(x)=\frac{1}{2 \sqrt{2 \pi} \sigma_{y} x} e^{-\frac{(\ln \sqrt{x})^{2}}{2 \sigma_{y}^{2}}} .
$$

The $p d f$ of $P_{t \max } r_{11}^{-\gamma} e^{2 \zeta_{1}(L)}$ is given by

$$
\begin{aligned}
& f_{P_{\text {inmax }} \gamma_{1:}^{-\gamma} e^{2 \zeta_{1}(L)}}(x) \\
& =\frac{1}{2 \sqrt{2 \pi} \sigma_{\zeta_{1}} x} e^{\frac{\left(\ln \left(\sqrt{x / P_{\max } \gamma_{1}^{-\gamma}}\right)\right)^{2}}{2 \sigma_{\zeta_{1}}^{2}}} .
\end{aligned}
$$


From equation (16), the $p d f$ of $P_{e r r}^{2}$ can be derived as

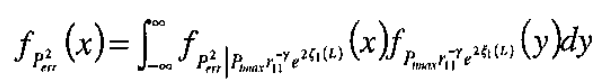

where

$$
\begin{aligned}
& f_{P_{r n}^{2} \mid P_{\text {max }} e^{2 \zeta_{1}(u)}}(x)
\end{aligned}
$$

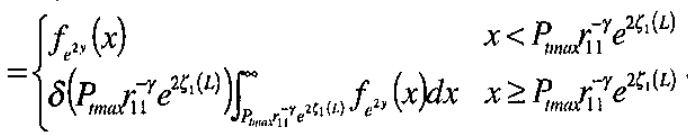

$$
\text { So }
$$

$$
\begin{aligned}
& f_{P_{e r t}^{2}}(x)=\int_{-\infty}^{+\infty}\left[f_{e^{2 y}}(x) U(y-x)\right. \\
& +\delta(x-y) \int_{y}^{\infty} f_{e^{2 y}}(x) d x \mid f_{P_{\max } x_{y 1}^{-y} e^{2 z_{1}(\alpha)}}(y) d y \\
& =f_{e^{2 y}}(x) \int_{-\infty}^{+\infty} U(y-x) f_{P_{\operatorname{man} x} x_{1}^{-y} e^{2 b_{1}(t)}}(y) d y \\
& +\int_{-\infty}^{+\infty} \delta(x-y) \int_{y}^{\infty} f_{e^{2 y}}(x) d x f_{P_{\text {man }} \gamma_{11}^{-\gamma} e^{2 \zeta_{1}(l)}}(y) d y
\end{aligned}
$$

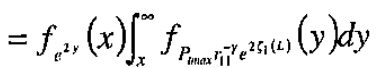

$$
\begin{aligned}
& +f_{P_{\max x} x_{1}^{-\gamma} e^{2 \xi_{1}(L)}}(x) \int_{x}^{\infty} f_{e^{2 y}}(y) d y
\end{aligned}
$$

Then, the $p d f$ of $P_{e r r}$ can be given by

$$
f_{P_{r r}}(x)=2 x f_{P_{r t}^{2}}\left(x^{2}\right) U(x) \text {. }
$$

The mean and standard derivation of $P_{e r r}$ is given by

$$
\bar{P}_{e r r}=\int_{0}^{\infty} x f_{P_{e r r}}(x) d x
$$

and

$$
\sigma_{P_{e r t}}=\left\{\int_{0}^{\infty}\left[x-E\left[f_{P_{r t r}}(x)\right]\right]^{2} f_{P_{r v}}(x) d x\right\}^{1 / 2} .
$$

By numerical integration, the mean and standard derivation of $P_{e r r}$ can be calculated from (31) and (32).

\section{Numerical Results}

The numerical results are shown in the following. Unless noted otherwise, the following parameters are assumed: the active users of one cell $K=20$, the standard derivation of shadowing $\sigma_{\zeta}=8 \mathrm{~dB}$, the path loss exponent $\gamma=4$, the information data rate $10 \mathrm{~Kb} / \mathrm{s}$, the speed of a mobile user $v=100 \mathrm{~km} / \mathrm{h}$, the carrier frequency $f_{0}=2 \mathrm{GHz} \quad$ (Dopplor frequency shift $\left.f_{d}=v / \lambda=185 \mathrm{~Hz}\right)$, the period for power estimation $L=60$ (or 60 bits), and the shadowing correlation distance $D=45 \mathrm{~m}$.

Fig. 2 shows the variation of the mean of powercontrol-error as a function of $r_{11} / R$ for two power limitation cases, $\quad P_{\text {tmax }} / P_{R}=5 d B \quad$ and
$P_{\text {tmax }} / P_{R}=2 d B$. It is shown from the figure that the mean of the power control error will decrease with the decrease of $P_{\text {tmax }}$. This will let the received signal level lower than the desired level and reduce the system capacity. The figure is also shown that the affects from transmit power limitation are not same for differently distributed mobile users. When a user near the base station, its transmit power to the base station is small and the affect from the power limitation can be neglected. In this case, the power control error is just from the measurement error. It is got from (18) and (23).

Fig. 3 shows the standard derivation of powercontrol-error as a function of $r_{11} / R$ for different $P_{\text {tmax }} / P_{R}$. It is shown that the standard derivation undergoes the same changing characteristic as that of the mean. However, we can't say the performance of the system will be improved because of the decrease of standard derivation with the decrease of $P_{t \max }$. This is because the mean is also decreased at the same time. In this case the decrease of the standard derivation will deteriorate the system performance more.

Fig. 4 shows the worst case system BER witl: the variation of $P_{\text {tnax }}$. It is shown from the figure that when $P_{\text {tmax }} / P_{R}<10 \mathrm{~dB}$, the BER will be affected significantly.

\section{Conclusions}

The impact of dynamic range to the open-loop power control error has been studied. From our assumed model, the following conclusions have been drawn:

(1) The exist of upper bound transmit power will reduce the mean and standard derivation of power control error. When a user near the base station, this affect will be insignificant.

(2) The system BER will decrease with the decrease of $P_{\max }$.

\section{Reference}

[1] Monk, L. B. Milstein, "Open-Loop Power Control Error in a Land Mobile Satellite System," IEEE J. Select. Areas Commun., vol.13, pp. 205-212, Feb. 1995

[2] R. .R. Gejji, "Forward-Link-Power Control in CDMA Cellular Systems," IEEE Trans. Veh. Technol., vol.41, pp532-536, Nov. 1992

[3] M. Gudmundson, "Correlation Model for Shadow Fading in Mobile Radio Systems," Electr. Letts., vol.27, pp. 2145-2146, Nov. 1991 
[4] R. H. Clarke, "A Statistical Theory of Mobile Radio Reception," Bell Syst. Tech. J., vol. 47, pp. 957-1000, July/Aug. 1968

[5] A. Papoulis, "Probability, Random Variables, and Stochastic Processes", McGraw-Hill, Inc., New York, 1991

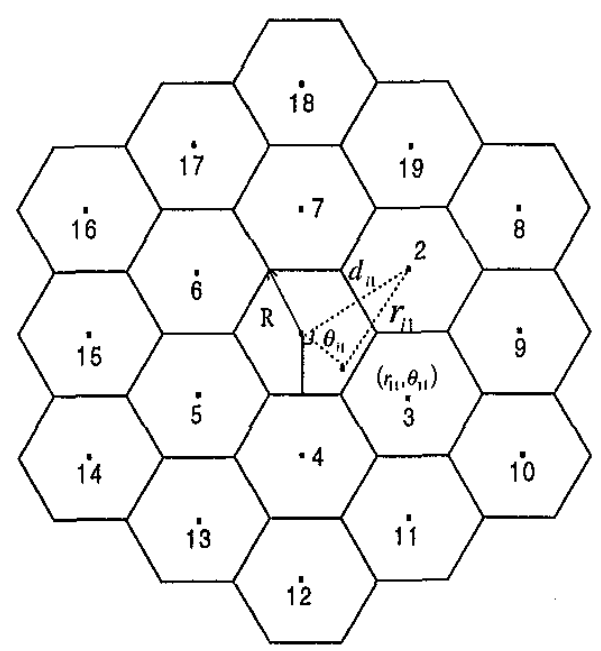

Fig.1 Downlink adjacent cell interference geometry

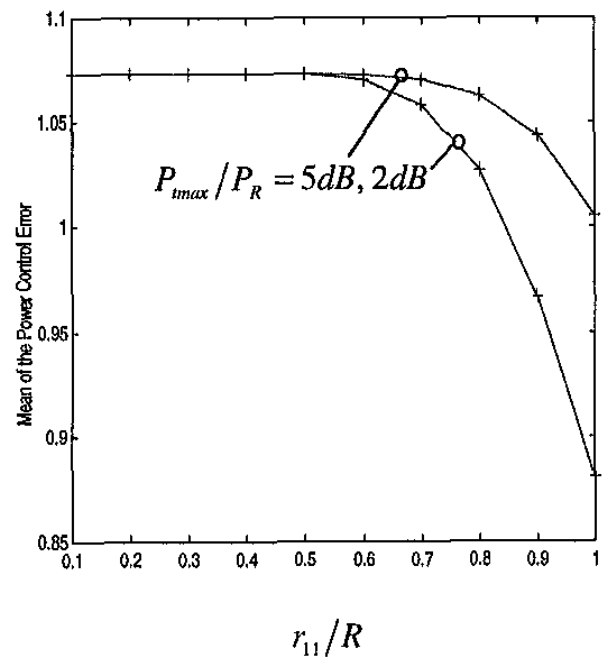

Fig. 2. Mean of the power control error as a function of $r_{11} / R$

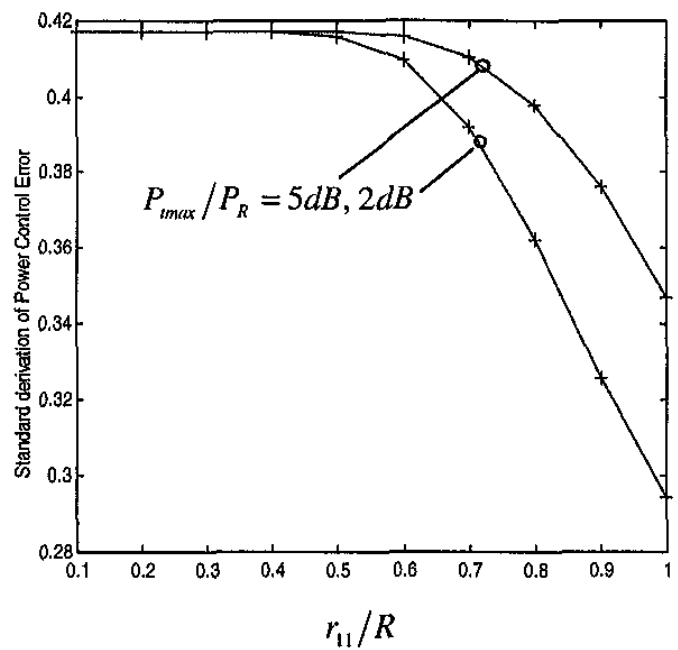

Fig. 3. Standard derivation of power control error as a function of $r_{11} / R$

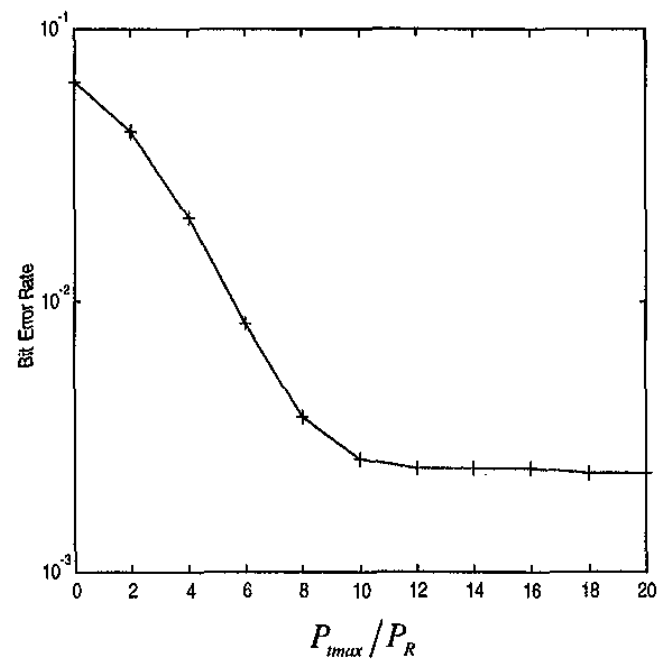

Fig. 4. The BER performance versus $P_{\text {tmax }} / P_{R}$ 